ORIGINAL ARTICLE

\title{
Making an IMPACT on emergency department flow: improving patient processing assisted by consultant at triage
}

\author{
J Terris, P Leman, N O'Connor, R Wood
}

Emerg Med J 2004;21:537-541. doi: 10.1136/emj.2002.003913

See end of article for authors' affiliations

.....................

Correspondence to: Dr J Terris, Emergency Department, St Thomas's Hospital, Lambeth Palace Road, London SE1 7EH, UK; jane.terris@gstt. sthames.nhs.uk

Accepted for publication 14 August 2003

\begin{abstract}
Objectives: To assess whether initial patient consult by senior clinicians reduces numbers of patients waiting to be seen as an indirect measure of waiting time throughout the emergency department (ED). Methods: An emergency medicine consultant and a senior ED nurse ( $G$ or F grade), known as the IMPACT team, staffed the triage area for four periods of four hours per week, Monday to Friday between 9 am to 5 pm for three months between December 2001 and February 2002 when staffing levels permitted. Patients normally triaged by a nurse in this area instead had an early consultation with the IMPACT team. Data were collected prospectively on all patients seen by the IMPACT team. The number of patients waiting to be seen (for triage, in majors and in minors) was assessed every two hours during the IMPACT sessions and at corresponding times when no IMPACT team was operational.

Results: There was an overall reduction in the number of patients waiting to be seen in the department from 18.3 to $5.5(p<0.0001)$ at formal two hourly assessments. The largest difference was seen in minors. Of the patients seen at triage by the IMPACT team, $48.9 \%$ were discharged home immediately after assessment and treatment. With the IMPACT team present, no patient waited more than four hours for initial clinical consult.

Conclusions: By using a senior clinical team for initial patient consultation, the numbers of patients waiting fell dramatically throughout the ED. Many patients can be effectively treated and discharged after initial consult by the IMPACT team.
\end{abstract}

$\mathrm{P}$ ressures on UK emergency departments (EDs) have never been greater to see more patients more quickly. By 2004, no patient should be waiting more than four hours in EDs from arrival to admission, transfer, or discharge, if government targets are to be met. ${ }^{1}$ Not only are adverse events and patient dissatisfaction related to length of wait to be seen by a clinician, ${ }^{2}$ but waiting times are a powerful clinical indicator for management purposes, and at government level are being used as a tool to determine the "star rating" of trusts.

There is a shortfall in senior staffing levels in UK EDs resulting in most initial patient consults being done by junior doctors $^{3}$ and most patients being nursed by comparatively junior nursing staff. Senior initial assessment can speed up treatment for all patients through good initial planning and appropriate use of investigations. ${ }^{3}$ Patient triage as commonly performed in the UK and abroad consists of patients undergoing an initial short nursing interview in order to be assigned a time frame of wait to see a clinician. Historically, patients assigned to categories 4 and 5 have had prolonged waits. For triage to be a key to clinical effectiveness in emergency care, it needs to direct the right patient to the right resources at the right time, and be strongly predictive of the severity of illness. ${ }^{4}$ Although Cooke ${ }^{5}$ in a pilot study found that the Manchester triage system detected the critically ill, the study was not powered to determine sensitivity and not designed to determine specificity. Other authors suggested that triage may fail to predict serious illness and need for admission ${ }^{6}$ and lead to an increase in waiting times for all triage categories. ${ }^{7}$ Among a group of patients prospectively assigned a low triage priority ( 4 on a 5 point scale) up to $33 \%$ may require hospital admission. ${ }^{8}$ There is also little provision within such systems to accommodate the experienced judgement of senior nurses, which may be largely intuitive and not be easily reproducible, but is none the less valid. ${ }^{9}$
Little validation has been carried out on the accuracy of currently performed triage practices in the UK. There is wide variation in both practice and training, and audit may be insufficient to ensure consistent application of assessment criteria in some EDs. ${ }^{3}$ It has been suggested that triage processes, as currently performed, should not be used to determine the timeliness of access to emergency care. ${ }^{10}$

We sought to replace the triage process of initial nurse consult to assign a waiting priority with a model of early senior clinician assessment, which has more recently been termed "see and treat". Such models have been previously assessed in the UK, ${ }^{11}$ Australia, ${ }^{12}$ and the USA, ${ }^{13}$ although the literature is sparse.

Our hypothesis was that an early senior clinician consult for patients, replacing the standard triage episode, would reduce numbers of patients waiting to be seen in the department and the overall patient journey would improve.

\section{METHODS}

The study was performed in an inner city teaching hospital ED, with an annual attendance of 108000 . Paediatric patients form $18 \%$ of the total attendances and there is a $20 \%$ admission rate. Medical staffing includes five consultant emergency physicians, 10 specialist registrars, and 25 junior SHOs. Primary care providers are rostered up to 50 hours cover per week. There are 124 WTE nursing staff, including eight emergency nurse practitioners.

Funding was allocated for senior clinicians (medical and nursing) to staff the triage area for 16 hours per week for three months. An emergency medicine consultant and a senior ED nurse ( $G$ or F grade) were chosen as the preferred team. The four hour sessions were scheduled between Monday to Friday, 9 am to $5 \mathrm{pm}$, and spread uniformly throughout the day and week. Comparison periods consisted of similar time blocks during the same three months when the IMPACT team were not working. 
After a brief verbal assessment on arrival by the "hello" nurse to ensure only that the patient was not too ill to give registration details before consultation, but without performing formal triage and assigning a triage category, the patient registered at reception and was then seen by the IMPACT team. The IMPACT team thus provided the first formal clinical assessment. The "hello" nurse performs an identical role before the usual triage when IMPACT is not operational. A focused history and screening examination, including vital signs where thought to be necessary, was performed by the consultant. Working flexibly with the IMPACT nurse, investigations and further treatment were expedited, admission or referral to another healthcare provider was facilitated, and where suitable, patients were discharged from triage.

To assess the efficacy of the IMPACT team on the ED we measured the number of patients waiting to be seen in each of the clinical areas studied (majors, minors, and triage). The resuscitation area was not assessed as all patients treated there are seen within five minutes. This was assessed at two hourly intervals (at $11 \mathrm{am}, 1 \mathrm{pm}, 3 \mathrm{pm}$, and $5 \mathrm{pm}$ ) throughout the study period (during either the IMPACT sessions or during control sessions). We believe that this provides a more pragmatic assessment of the utility of a service such as IMPACT, (that is, how many people are there in the queue) rather than the overall mean waiting time per person.

Data were collected prospectively on the characteristics of patients seen by the IMPACT team, and on the interventions performed by the team and subsequent disposition.

All patients seen by the IMPACT team were asked to fill in an anonymous patient satisfaction survey at the end of their ED episode of care (whether in triage or elsewhere in the department). They were asked whether the waiting time was longer, shorter, or the same as expected and whether they were; unsatisfied, satisfied, or very satisfied with their episode of care.

In an attempt to gauge any adverse outcomes, a telephone follow up survey was performed. This was one week after the consultation for half of the patients who were discharged direct from triage, by an investigator blinded to the initial consultation. Patients were asked whether they had made any unscheduled visits to a healthcare provider since discharge from ED for the same condition. The sample was selected by using the hospital record number and choosing only even numbers to follow up.
Data were analysed using SYSTAT software (version 10). Statistical analyses using $t$ tests were performed on continuous data and Mann-Whitney $U$ tests for nonnormally distributed data. We used $\chi^{2}$ tests for tabulated data.

\section{RESULTS}

Data forms were obtained for a total of 378 patients triaged by the team, during 23 sessions ( 92 hours) of IMPACT operation. Of the 23 IMPACT sessions only 20 had complete data for all three departmental areas recorded at the two hourly assessments, a total of 120 recordings. In the control two hourly assessments there were 22 sessions where the data were complete (132 records). These fully completed datasets were analysed further.

Nearly two thirds of patients (see table 1) presenting had an illness or injury that was at least one day old. Patients waited a mean of 16 to 20 minutes from registration until they were be seen by the IMPACT team. The median time was 13 minutes, and it was thought that the mean time had been increased by patients who were not in the waiting room when called for a number of reasons, and had then returned. Additionally, the time to IMPACT consult data was negatively affected by the backlog of patients waiting to be assessed at the start of each IMPACT session.

While the mean duration of assessment and treatment was 7.8 minutes, this was considerably increased by cases where a radiograph was ordered (mean 28.5 minutes) and subsequently reviewed by the IMPACT doctor at triage. Patients who had no radiological review required just over six minutes for assessment and treatment.

Just over one third $(35.6 \%)$ of the patients had standard triage observations measured (such as blood pressure, pulse rate, respiratory rate, etc) and only $9.5 \%$ required other simple investigations such as urine analysis or an ECG (see table 2). Nearly one fifth of patients had a radiograph ordered at triage, some of these were subsequently reviewed by the IMPACT doctor as part of the primary assessment (as well as review of GP requested radiographs). Of the patients who received POM (prescription only) medications (9.0\%), most were for either antibiotics or non-steroidal anti-inflammatory drugs. The IMPACT team was able to discharge nearly $50 \%$ of patients home directly after IMPACT consult. A further $6.9 \%$ were sent to a more appropriate clinic or to a scheduled GP appointment.

Table 1 Timings of illness duration and IMPACT triage process

\begin{tabular}{|c|c|c|c|c|c|}
\hline Parameter & $\begin{array}{l}\text { Number of complete } \\
\text { records available }\end{array}$ & Range & Median & Mean & $95 \% \mathrm{Cl}$ of mean \\
\hline \multicolumn{6}{|c|}{ Time from registration to IMPACT triage beginning (min) } \\
\hline $\begin{array}{l}\text { Overall } \\
\text { by diagnostic group }\end{array}$ & 355 & $0-213$ & 13 & 18.4 & 16.3 to 20.6 \\
\hline Injury & 206 & $0-213$ & 13 & 18.1 & 15.3 to 21.0 \\
\hline Illness & 132 & $1-170$ & 13 & 18.9 & 15.4 to 22.5 \\
\hline Not coded & 17 & $5-41$ & 18 & 18.0 & 13.0 to 23.0 \\
\hline \multicolumn{6}{|c|}{ Time from IMPACT triage beginning to IMPACT triage completed (min) } \\
\hline \multicolumn{5}{|l|}{ By diagnostic group } & 6.7 to 9.0 \\
\hline injury & 166 & $0-88$ & 5 & 9.01 & 7.3 to 10.7 \\
\hline illness & 114 & $0-30$ & 5 & 5.78 & 5.0 to 6.5 \\
\hline not coded & 10 & $1-65$ & 5 & 12.3 & 0 to 26.2 \\
\hline \multicolumn{6}{|c|}{ By radiological review at triage } \\
\hline no & 268 & $0-65$ & 5 & 6.2 & 5.5 to 6.8 \\
\hline yes & 22 & $5-88$ & 27 & 28.5 & 19.3 to 37.7 \\
\hline \multicolumn{6}{|c|}{ Duration of illness/injury $(n=368)$} \\
\hline In days (if $\geqslant 1$ day) & 243 & $1-750$ & 3 & 30.2 & 16.5 to 43.9 \\
\hline In hours (if $<1$ day) & 125 & $0-17$ & 2 & 3.09 & 2.5 to 3.6 \\
\hline
\end{tabular}


Table 2 Interventions and outcome during IMPACT triage

\begin{tabular}{|c|c|c|c|}
\hline Intervention & Number (total $n=378$ ) & Proportion (\%) & $95 \% \mathrm{Cl}$ \\
\hline \multicolumn{4}{|l|}{ Vital signs measured } \\
\hline None recorded & 243 & 64.3 & 58.4 to 69.7 \\
\hline Yes & 135 & 35.6 & 30.1 to 41.3 \\
\hline \multicolumn{4}{|l|}{ Simple investigation* } \\
\hline None recorded & 342 & 90.5 & 86.4 to 93.5 \\
\hline Yes & 36 & 9.5 & 6.4 to 13.4 \\
\hline \multicolumn{4}{|l|}{ Radiograph request } \\
\hline None recorded & 307 & 81.2 & 75.8 to 85.7 \\
\hline Extremity & 59 & 15.6 & 11.3 to 20.5 \\
\hline Non-extremity & 12 & 3.2 & 1.3 to 6.0 \\
\hline \multicolumn{4}{|l|}{ Procedures performed } \\
\hline None recorded & 325 & 86.0 & 81.4 to 89.7 \\
\hline Yes & 53 & 14 & 10.2 to 18.4 \\
\hline \multicolumn{4}{|l|}{ Drugs given at triage } \\
\hline None recorded & 342 & 90.5 & 85.7 to 93.8 \\
\hline Simple analgesia & 9 & 2.4 & 0.8 to 5.1 \\
\hline POM analgesia & 21 & 5.6 & 2.9 to 9.2 \\
\hline Other POM & 5 & 1.3 & 0.2 to 3.6 \\
\hline$>$ lof above & 1 & 0.3 & 0.0 to 1.8 \\
\hline \multicolumn{4}{|l|}{ Prescriptions written } \\
\hline None recorded & 307 & 81.4 & 75.8 to 86.1 \\
\hline POM & 34 & 9.0 & 5.6 to 13.2 \\
\hline Advise purchase of OTC & 35 & 9.3 & 5.8 to 13.5 \\
\hline POM and OTC & 1 & 0.3 & 0.0 to 1.8 \\
\hline Disposition & 377 & & \\
\hline ED & 100 & 26.5 & 20.9 to 32.4 \\
\hline Home & 185 & 48.9 & 42.3 to 55.4 \\
\hline Primary care or other clinic & 26 & 6.9 & 3.9 to 10.7 \\
\hline Unknown & 67 & 17.7 & 13.0 to 23.0 \\
\hline
\end{tabular}

Analysis of the three areas of the department where patients might be waiting to see a doctor or emergency nurse practitioner (ENPs in minors only) showed considerable differences (table 3 ). This was statistically significant in each area, but most noticeable in "minors" (mean difference of 8.8 patients less waiting to be seen; (95\% CI 6.7 to 10.9). There was an overall reduction from 18.3 to 5.5. (mean difference 12.8 ; $95 \%$ CI 10.2 to 15.4 ) patients waiting in the whole department at any one time. Figure 1 shows the change in numbers waiting to be seen.

At each of the two hourly assessments the presence of any patients waiting more than four hours to see a doctor was recorded. Of the two hourly assessments (120) where IMPACT was functioning no assessments recorded any waits of this length. Conversely when the assessments occurred without IMPACT functioning (132) there were seven occasions where one or more patients had been waiting more than four hours to see the doctor, this was significant $\left(\chi^{2}\right.$; $6.2, \mathrm{p}=0.01$ ).

A total of 185 patients were discharged home directly after IMPACT consult and all reported being very satisfied with their episode of care and that they had waited much less time than expected. Of the 87 patients suitable for telephone follow up, only 45 could be contacted because of difficulties inherent in following up inner city ED patient populations, and of these no adverse events or need for unscheduled care were reported.

\section{DISCUSSION}

Doctors are not commonly part of the initial patient consult in the UK. There are no previous studies of the effect of emergency physician or of senior nurse initial consult on numbers of ED patients waiting to be seen, though other parameters have been measured. Previous studies have examined the effect on ED waiting time to be seen by a doctor by using physician triage ${ }^{12}$ and of ED length of stay after consultant triage. ${ }^{11} 13$ Findings have included reduced lengths of stay and waiting times, but the process was not thought to be sustainable without additional staffing resources. ${ }^{12} 13$

In our study the effect of the additional resources was not directly measured, we acknowledge that increasing the overall staffing complement by a consultant plus a senior nurse would be expected to give some improvement in the parameters measured whether IMPACT was occurring or not. Although our staffing levels are generally high in comparison with national standards, current resources within our department are inadequate for IMPACT to operate on a full time basis, but where staffing levels permit, we feel it to be a useful tool for managing flow at times of peak attendance.

Table 3 Effect of IMPACT on the number of patients waiting to be seen, overall and by area

\begin{tabular}{|c|c|c|c|c|}
\hline \multirow[b]{2}{*}{ Area of department } & \multicolumn{2}{|c|}{ Mean (SD) number of patients waiting } & \multirow{2}{*}{$\begin{array}{l}\text { Mean difference } \\
(95 \% \mathrm{Cl})\end{array}$} & \multirow[b]{2}{*}{$\mathrm{p}$ Value } \\
\hline & No IMPACT & With IMPACT & & \\
\hline $\begin{array}{l}\text { Overall } \\
\text { Triage } \\
\text { Minors } \\
\text { Majors }\end{array}$ & $\begin{array}{l}18.3(7.4) \\
2.7(2.1) \\
13.0(5.6) \\
3.0(1.5)\end{array}$ & $\begin{array}{l}5.5(4.2) \\
0.8(1.2) \\
4.1(3.8) \\
0.7(0.6)\end{array}$ & $\begin{array}{l}12.8(10.2 \text { to } 15.4) \\
1.9(1.1 \text { to } 2.6) \\
8.8(6.7 \text { to } 10.9) \\
2.3(1.8 \text { to } 2.8)\end{array}$ & $\begin{array}{l}<0.0001 \\
<0.0001 \\
<0.0001 \\
<0.0001\end{array}$ \\
\hline
\end{tabular}




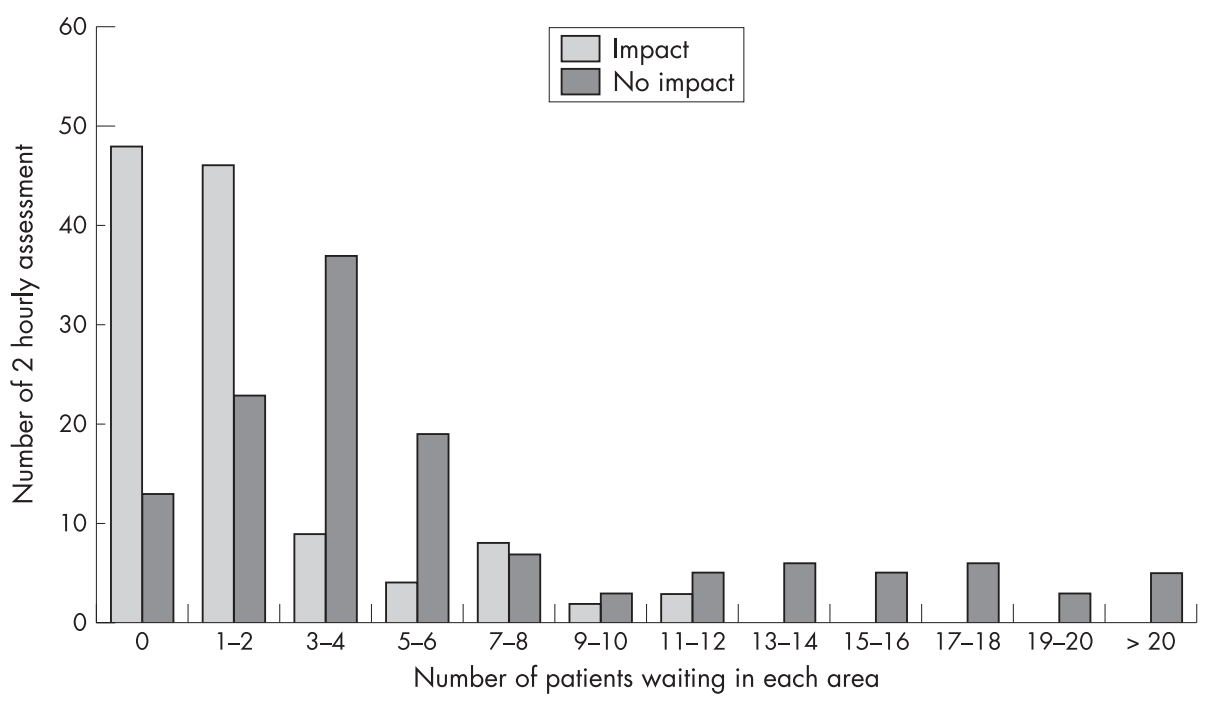

Figure 1 Two hourly assessments of numbers of patients waiting to be seen.

Clearly in departments without our overall level of staffing it would be difficult to provide an IMPACT type service, let alone sustain it for any period of time. Even with the extra staff only 23 of the intended number of 48 IMPACT sessions could be completed because of staffing shortages on some days, and although our intention was that the IMPACT team should have no other duties during that time, this did not always happen.

The direct and indirect costs of such a service would be considerable and this pilot project was not designed to provide a formal cost effectiveness analysis. It may well be that while IMPACT can provide us with the tangible benefits shown in this study the money required to even partially fund such a service could produce even greater benefits elsewhere within the ED, we have not measured such opportunity costs in this paper.

We believe, however, that by using the IMPACT team for the initial consult, other benefits ensue, such as early discharge for almost half the patients seen after IMPACT consult, fewer patients needing to wait in the waiting room, and improved journeys for the patients not discharged after the initial consult, including the opportunity to detect serious disorder at an early stage.

While our brief patient satisfaction survey was overwhelmingly positive we did not obtain an adequate comparison sample during the non-IMPACT times from patients triaged in the normal manner. Previous studies have found waiting times to be seen in ED to be a major determinant of patient satisfaction. ${ }^{2}$ The median time to be seen by the IMPACT team was longer than we expected. This was partly attributable to patients not being in the waiting room when called, but mostly because of already existing long waits for triage when the IMPACT team started their four hour session.

We sought to facilitate earlier patient consultation by senior clinicians at triage and chose to measure numbers of patients waiting in three areas of the department as a possible predictor of waiting time, and hence patient satisfaction, ${ }^{2}$ and a possible index of workload for ED staff. We chose the particular skill mix of the IMPACT team to attempt to produce the most senior experienced assessment possible on arrival for patients. Few vital signs were measured at triage by nurse or consultant, which reflects the authors' experience that vital signs do not play a significant part in senior clinician triage decisions for most ambulant patients, but may be important in a small number of patients. Previous authors have commented on the limited role of vital signs in changing nurse triage decisions ${ }^{14}$ as they probably rely less on actual recorded vital signs and more on history, other visual cues, and physical findings.

No adverse events or unscheduled consults with healthcare professionals for the same problem were detected in the sample able to be followed up after discharge from triage, although for various reasons only 45 responses of a total 87 were obtained (usually because of non-availability of the patient by telephone during the day). This has been a finding in previous ED studies. ${ }^{15}$

In addition to reduced numbers of patients waiting in the $\mathrm{ED}$, other benefits were observed that were more difficult to measure, including an opportunity for all ambulant patients to be assessed by a consultant at the earliest opportunity, a sense of improved management of patient flow, and optimising use of departmental skill mix. The nursing staff commented that not only were they less stressed, but that the patients were also less verbally demanding and less likely to be aggressive. IMPACT was felt to be demanding by the clinicians performing the initial assessments. It was not possible to continue unless clinical time devoted to IMPACT was protected by having equivalent senior clinicians responsible for patients in other areas of the ED. For IMPACT to continue requires additional senior clinicians, an observation also made by other authors. ${ }^{12} 13$

We believe the IMPACT team model gives the opportunity to simultaneously "see and treat" all ambulant patients, effectively stream those patients requiring more prolonged consultations and expedite investigations and inpatient referral processes. It comes at a considerable cost however and can only function with a separate and additional consultant and nurse from those who are staffing the department and assessing the non-ambulant patients.

Best evidence on the IMPACT model would be provided by a prospective randomised, controlled trial. Our essentially observational study will have suffered from the Hawthorne effect and the consultants and nurses involved in the project put in considerable effort to see the model through, this is unlikely to be sustained if the model was used as standard care. Furthermore, we would like to see future work assessing an IMPACT type team on non-ambulant ED patients.

\section{Authors' affiliations}

J Terris, P Leman, N O'Connor, R Wood, Emergency Department, St Thomas's Hospital, London, UK 


\section{REFERENCES}

1 Department of Health. The NHS plan. London: Department of Health, 2000.

2 Holden D, Smart S. Adding value to the patient experience in emergency medicine: What features of the emergency department visit are most important to patients? Emergency Medicine 1999;1 1:3-8.

3 Audit Commission. By accident or design - improving A\&E services in England or Wales. London: HMSO, 1996.

4 Fernandes C, Wuerz R, Clark S, et al. How reliable is emergency department triage? Ann Emerg Med 1999;34:141-7.

5 Cooke MW, Jinks S. Does the Manchester triage system detect the critically ill? J Accid Emerg Med 1999;16:179-81.

6 Brillman J, Doezema D, Tandberg D, et al. Triage: limitations in predicting need for emergent care and hospital admission. Ann Emerg Med 1996:27:493-500.

7 George S, Read S, Westlake L, et al. Evaluation of nurse triage in a British accident and emergency department. BMJ 1992;304:876-8.
8 Dent A, Rofe G, Sansom G. Which triage category patients die in hospital after being admitted through emergency departments? A study in one teaching hospital. Emergency Medicine 1999;11:68-71.

9 Mackway-Jones K, ed. Emergency triage. London: BMJ Books, 1997.

10 Wuerz R, Fernandes C, Alarcon J. Inconsistency of emergency department triage. Ann Emerg Med 1998;32:431-5.

11 Redmond AD, Buxton N. Consultant triage of minors cases in an accident and emergency department. Arch Emerg Med 1993;10:328-30.

12 Grant S, Spain D, Green D. Rapid assessment team reduces waiting time. Emergency Medicine 1999;11:72-7.

13 Sirous N, Partovi S, Nelson B, et al. Faculty triage shortens emergency department length of stay. Acad Emerg Med 2001;10:990-5.

14 Cooper RJ, Schriger DL, Flaherty HL, et al. Effect of vital signs on triage decisions. Ann Emerg Med 2002;39:223-32.

15 Derlet R, Kinser D, Ray L, et al. Prospective identification and triage of nonemergency patients out of an emergency department: a 5 year study. Ann Emerg Med 1995;25:215-23. 